\title{
PASCAL-XSC \\ New Concepts for Scientific Computation and Numerical Data Processing
}

\author{
R. Hammer, M. Neaga, and D. Ratz
}

The new programming language PASCAL-XSC is presented with an emphasis on the new concepts for scientific computation and numerical data processing of the PASCAL-XSC compiler. PASCAL-XSC is a universal PASCAL extension with extensive standard modules for scientific computation. It is available for personal computers, workstations, mainframes and supercomputers by means of an implementation in $\mathrm{C}$.

By using the mathematical modules of PASCAL-XSC, numerical algorithms which deliver highly accurate and automatically verified results can be programmed easily. PASCAL-XSC simplifies the design of programs in engineering scientific computation by modular program structure, user-defined operators, overloading of functions, procedures, and operators, functions and operators with arbitrary result type, dynamic arrays, arithmetic standard modules for additional numerical data types with operators of highest accuracy, standard functions of high accuracy and exact evaluation of expressions.

The most important advantage of the new language is that programs written in PASCAL-XSC are easily readable. This is due to the fact that all operations, even those in the higher mathematical spaces, have been realized as operators and can be used in conventional mathematical notation.

In addition to PASCAL-XSC a large number of numerical problem-solving routines with automatic result verification are available. The language supports the development of such routines.

\section{Introduction}

These days, the elementary arithmetic operations on electronic computers are usually approximated by floating-point operations of highest accuracy. In particular, for any choice of operands this means that the computed result coincides with the rounded exact result of the operation. See the IEEE Arithmetic Standard [3] as an example. This arithmetical standard also requires the four basic arithmetic operations,,$+- *$, and / with directed roundings. A large number of processors already on the market provide these operations. So far, however, no common programming language allows access to them.

On the other hand, there has been a noticeable shift in scientific computation from general purpose computers to vector and parallel computers. These so-called

Scientific Computing with Automatic Result Verification
15 Copyright (c) 1993 by Academic Press, Inc. All rights of reproduction in any form reserved.

ISBN 0-12-044210-8 
super-computers provide additional arithmetic operations such as "multiply and add" and "accumulate" or "multiply and accumulate" (see [10]). These hardware operations should always deliver a result of highest accuracy, but as of yet, no processor which fulfills this requirement is available. In some cases, the results of numerical algorithms computed on vector computers are totally different from the results computed on a scalar processor (see [13],[31]).

Continuous efforts have been made to enhance the power of programming languages. New powerful languages such as ADA have been designed, and enhancement of existing languages such as FORTRAN is in constant progress. However, since these languages still lack a precise definition of their arithmetic, the same program may produce different results on different processors.

PASCAL-XSC is the result of a long-term venture by a team of scientists to produce a powerful tool for solving scientific problems. The mathematical definition of the arithmetic is an intrinsic part of the language, including optimal arithmetic operations with directed roundings which are directly accessable in the language. Further arithmetic operations for intervals and complex numbers and even vector/matrix operations provided by precompiled arithmetical modules are defined with maximum accuracy according to the rules of semimorphism (see [25]).

\section{The Language PASCAL-XSC}

PASCAL-XSC is an extension of the programming language PASCAL for $\underline{\text { Scientific }}$ Computation. A first approach to such an extension (PASCAL-SC) has been available since 1980. The specification of the extensions has been continuously improved in recent years by means of essential language concepts, and the new language PASCAL-XSC [20],[21] was developed. It is now available for personal computers, workstations, mainframes, and supercomputers by means of an implementation in C. PASCAL-XSC contains the following features:

- Standard PASCAL

- Universal operator concept (user-defined operators)

- Functions and Operators with arbitrary result type

- Overloading of procedures, functions and operators

- Module concept

- Dynamic arrays

- Access to subarrays

- String concept

- Controlled rounding

- Optimal (exact) scalar product 
- Standard type dotprecision (a fixed point format to cover the whole range of floating-point products)

- Additional arithmetic standard types such as complex, interval, rvector, rmatrix etc.

- Highly accurate arithmetic for all standard types

- Highly accurate standard functions

- Exact evaluation of expressions (\#-expressions)

The new language features, developed as an extension of PASCAL, will be discussed in the following sections.

\subsection{Standard Data Types, Predefined Operators, and Func- tions}

In addition to the data types of standard PASCAL, the following numerical data types are available in PASCAL-XSC:

$\begin{array}{llll}\text { interval complex } & \text { cinterval } & \\ \text { rvector } & \text { cvector } & \text { ivector } & \text { civector } \\ \text { rmatrix cmatrix } & \text { imatrix } & \text { cimatrix }\end{array}$

where the prefix letters $r, i$, and $c$ are abbreviations for $\underline{r} e a l$, interval, and $\underline{c}$ mplex. So cinterval means complex interval and, for example, cimatrix denotes complex interval matrices, whereas rvector specifies real vectors. The vector and matrix types are defined as dynamic arrays and can be used with arbitrary index ranges.

A large number of operators are predefined for theses types in the arithmetic modules of PASCAL-XSC (see section 2.8). All of these operators deliver results with maximum accuracy. In Table 1 the 29 predefined standard operators of PASCAL$\mathrm{XSC}$ are listed according to priority.

\begin{tabular}{|c|c|c|}
\hline Type & Priority & Operators \\
\hline monadic & $\begin{array}{c}3 \\
\text { (highest) }\end{array}$ & monadic + , monadic - , not \\
\hline multiplicative & 2 & $\begin{array}{l}\text { and, div, mod } \\
*, *<, *>, /, /<, />, * *\end{array}$ \\
\hline additive & 1 & $\begin{array}{l}\text { or } \\
+,+<,+>,-,-<,->,+*\end{array}$ \\
\hline relational & $\begin{array}{c}0 \\
\text { (lowest) }\end{array}$ & $\begin{array}{l}\text { in } \\
=,<>,<=,<,>=,>,><\end{array}$ \\
\hline
\end{tabular}

Table 1: Precedence of the Built-in Operators 
Compared to standard PASCAL, there are 11 new operator symbols. These are the operators $0<$ and $o>, \circ \in\{+,-, *, /\}$ for operations with downwardly and upwardly directed rounding and the operators $* *,+*,><$ needed in interval computations for the intersection, the convex hull, and the disconnectivity test.

Tables 2 and 3 show all predefined arithmetic and relational operators in connection with the possible combinations of operand types.

\begin{tabular}{|c|c|c|c|c|c|c|}
\hline $\begin{array}{l}\text { right } \\
\text { left } \\
\text { operand }\end{array}$ & $\begin{array}{l}\text { integer } \\
\text { real } \\
\text { complex }\end{array}$ & $\begin{array}{l}\text { interval } \\
\text { cinterval }\end{array}$ & $\begin{array}{l}\text { rvector } \\
\text { cvector }\end{array}$ & $\begin{array}{l}\text { ivector } \\
\text { civector }\end{array}$ & $\begin{array}{l}\text { rmatrix } \\
\text { cmatrix }\end{array}$ & $\begin{array}{l}\text { imatrix } \\
\text { cimatrix }\end{array}$ \\
\hline monadic $\left.^{1}\right)$ &,+- &,+- &,+- &,+- &,+- &,+- \\
\hline $\begin{array}{c}\text { integer } \\
\text { real } \\
\text { complex }\end{array}$ & $\begin{array}{c}\left.{ }^{2}\right) \\
\circ, 0<, \circ> \\
+*\end{array}$ & $\begin{array}{c}+,-, *, / \\
+*\end{array}$ & $*, *<, *>$ & $*$ & $*, *<, *>$ & $*$ \\
\hline $\begin{array}{l}\text { interval } \\
\text { cinterval }\end{array}$ & $\begin{array}{c}+,-, *, / \\
+*\end{array}$ & $\begin{array}{c}+,-, *, / \\
\quad+*, * *\end{array}$ & $*$ & $*$ & $*$ & $*$ \\
\hline $\begin{array}{l}\text { rvector } \\
\text { cvector }\end{array}$ & $\begin{array}{c}*, *<, *> \\
/, /<, />\end{array}$ & $*, /$ & $\begin{array}{c}, 0<, \circ> \\
+*\end{array}$ & $\begin{array}{c}{ }^{4} \\
+,-, *, \\
+*\end{array}$ & & \\
\hline $\begin{array}{l}\text { ivector } \\
\text { civector }\end{array}$ & $*, /$ & $*, /$ & $\begin{array}{c}{ }^{4} \\
+,-, * \\
+*\end{array}$ & $\begin{array}{r}\left.{ }^{4}\right) \\
+,-, *, \\
+*, * *\end{array}$ & & \\
\hline $\begin{array}{l}\text { rmatrix } \\
\text { cmatrix }\end{array}$ & $\begin{array}{c}*, *<, *> \\
/, /<, />\end{array}$ & $*, /$ & $*, *<, *>$ & $*$ & $\begin{array}{c}\left.0,0<, \circ>^{3}\right) \\
+*\end{array}$ & $\begin{array}{c}\left.{ }^{4}\right) \\
+,-, *, \\
+*\end{array}$ \\
\hline $\begin{array}{l}\text { imatrix } \\
\text { cimatrix }\end{array}$ & $*, /$ & $*, /$ & $*$ & $*$ & $\begin{array}{c}{ }^{4} \\
+,-,{ }^{*} \\
+*\end{array}$ & $\begin{array}{r}{ }^{4} \\
+,-, *, \\
+*, * *\end{array}$ \\
\hline
\end{tabular}

1) The operators of this row are monadic (i.e. there is no left operand).

2) $\circ \in\{+,-, *, /\}$

$3) \circ \in\{+,-, *\}$, where $*$ denotes the scalar or matrix product.

4 ) denotes the scalar or matrix product.

$+*$ : Interval hull

** : Interval intersection

Table 2: Predefined Arithmetical Operators 
Compared with standard PASCAL, PASCAL-XSC provides an extended set of mathematical standard functions (see table 4). These functions are available for the types real, complex, interval, and cinterval with a generic name and deliver a result of maximum accuracy. The functions for the types complex, interval, and cinterval are provided in the arithmetic modules of PASCAL-XSC.

\begin{tabular}{|c|c|c|c|c|c|c|}
\hline $\begin{array}{l}\text { right } \\
\text { left } \\
\text { operand }\end{array}$ & $\begin{array}{c}\text { integer } \\
\text { real } \\
\text { complex }\end{array}$ & $\begin{array}{l}\text { interval } \\
\text { cinterval }\end{array}$ & $\begin{array}{l}\text { rvector } \\
\text { cvector }\end{array}$ & $\begin{array}{l}\text { ivector } \\
\text { civector }\end{array}$ & $\begin{array}{l}\text { rmatrix } \\
\text { cmatrix }\end{array}$ & $\begin{array}{l}\text { imatrix } \\
\text { cimatrix }\end{array}$ \\
\hline $\begin{array}{c}\text { integer } \\
\text { real } \\
\text { complex }\end{array}$ & $\begin{array}{l}=,<>, \\
<=,<, \\
>=,>\end{array}$ & $\begin{array}{c}\text { in } \\
=,<>\end{array}$ & & & & \\
\hline $\begin{array}{l}\text { interval } \\
\text { cinterval }\end{array}$ & $=,<>$ & $\begin{array}{c}\text { in },><, \\
=,<> \\
<=,<, \\
>=,>\end{array}$ & & & & \\
\hline $\begin{array}{l}\text { rvector } \\
\text { cvector }\end{array}$ & & & $\begin{array}{l}=,<>, \\
<=,<, \\
>=,>\end{array}$ & $\begin{aligned} & \text { in } \\
= & ,<>\end{aligned}$ & & \\
\hline $\begin{array}{l}\text { ivector } \\
\text { civector }\end{array}$ & & & $=,<>$ & $\begin{array}{l}\left.\text { in, }><{ }^{1}\right) \\
=,<>, \\
<=,<, \\
>=,>\end{array}$ & & \\
\hline $\begin{array}{l}\text { rmatrix } \\
\text { cmatrix }\end{array}$ & & & & & $\begin{array}{l}=,<>, \\
<=,<, \\
>=,>\end{array}$ & $\begin{aligned} & \text { in } \\
= & ,<>\end{aligned}$ \\
\hline $\begin{array}{l}\text { imatrix } \\
\text { cimatrix }\end{array}$ & & & & & $=,<>$ & $\begin{array}{l}\text { in, }><\text {, } \\
=,<>, \\
<=,<, \\
>=,>\end{array}$ \\
\hline
\end{tabular}

1) The operators $<=$ and $<$ denote the "subset" relations, $>=$ and $>$ denote the "superset" relations.

$><$ : Test on disconnectivity for intervals

in : Test on membership of a point in an interval or test on strict enclosure of an interval in the interior of an interval

Table 3: Predefined Relational Operators 


\begin{tabular}{|c|c|c|c|}
\hline & Function & Generic Name & Argument Type \\
\hline 1 & Absolute Value & abs & * \\
\hline 2 & Arc Cosine & $\arccos$ & * \\
\hline 3 & Arc Cotangent & $\operatorname{arccot}$ & * \\
\hline 4 & Inverse Hyperbolic Cosine & $\operatorname{arcosh}$ & * \\
\hline 5 & Inverse Hyperbolic Cotangent & arcoth & * \\
\hline 6 & Arc Sine & $\arcsin$ & * \\
\hline 7 & Arc Tangent & $\arctan$ & * \\
\hline 8 & Inverse Hyperbolic Sine & $\operatorname{arsinh}$ & $*$ \\
\hline 9 & Inverse Hyperbolic Tangent & $\operatorname{artanh}$ & * \\
\hline 10 & Cosine & $\cos$ & $*$ \\
\hline 11 & Cotangent & $\cot$ & $*$ \\
\hline 12 & Hyperbolic Cosine & $\cosh$ & $*$ \\
\hline 13 & Hyperbolic Cotangent & $\operatorname{coth}$ & $*$ \\
\hline 14 & Exponential Function & $\exp$ & * \\
\hline 15 & Power Function (Base 2) & $\exp 2$ & * \\
\hline 16 & Power Function (Base 10) & $\exp 10$ & $*$ \\
\hline 17 & Natural Logarithm (Base $e$ ) & $\ln$ & * \\
\hline 18 & Logarithm (Base 2) & $\log 2$ & * \\
\hline 19 & Logarithm (Base 10) & $\log 10$ & * \\
\hline 20 & Sine & $\sin$ & $*$ \\
\hline 21 & Hyperbolic Sine & $\sinh$ & * \\
\hline 22 & Square & sqr & * \\
\hline 23 & Square Root & sqrt & $*$ \\
\hline 24 & Tangent & $\tan$ & $*$ \\
\hline 25 & Hyperbolic Tangent & $\tanh$ & $*$ \\
\hline
\end{tabular}

Table 4: Mathematical Standard Functions (* includes the types integer, real, complex, interval, and cinterval)

Besides the mathematical standard functions, PASCAL-XSC provides the necessary type transfer functions intval, inf, sup, compl, re, and im for conversion between the numerical data types (for scalar and array types). 


\subsection{The General Operator Concept}

By a simple example of interval addition, the advantages of a general operator concept are demonstrated. In the absence of userdefined operators, there are two ways to implement the addition of two variables of type interval declared by

type interval $=$ record inf, sup: real; end;

One can use a procedure declaration

procedure intadd(a,b: interval; var c: interval);

begin

$$
\begin{aligned}
& \text { c.inf }:=\text { a.inf }+<\text { b.inf; } \\
& \text { c.sup }:=\text { a.sup }+>\text { b.sup }
\end{aligned}
$$

end;

\begin{tabular}{|c|c|}
\hline mathematical notation & corresponding program statements \\
\hline$z:=a+b+c+d$ & intadd $(\mathrm{a}, \mathrm{b}, \mathrm{z}) ;$ \\
& $\operatorname{intadd}(\mathrm{z}, \mathrm{c}, \mathrm{z}) ;$ \\
& intadd $(\mathrm{z}, \mathrm{d}, \mathrm{z}) ;$ \\
\hline
\end{tabular}

or a function declaration (only possible in PASCAL-XSC, not in standard PASCAL)

function intadd(a,b: interval): interval;

begin

intadd.inf $:=$ a.inf $+<$ b.inf;

intadd.sup $:=$ a.sup $+>$ b.sup

end;

\begin{tabular}{|c|c|}
\hline mathematical notation & corresponding program statement \\
\hline$z:=a+b+c+d$ & $\mathrm{z}:=\operatorname{intadd}(\operatorname{intadd}(\operatorname{intadd}(\mathrm{a}, \mathrm{b}), \mathrm{c}), \mathrm{d}) ;$ \\
\hline
\end{tabular}

In both cases the description of the mathematical formulas looks rather complicated. By comparison, if one implements an operator in PASCAL-XSC

operator $+(\mathrm{a}, \mathrm{b}$ : interval) intadd: interval;

begin

intadd.inf $:=$ a.inf $+<$ b.inf;

intadd.sup $:=$ a.sup $+>$ b.sup

end; 


\begin{tabular}{|c|c|}
\hline mathematical notation & corresponding program statement \\
\hline$z:=a+b+c+d$ & $\mathrm{z}:=\mathrm{a}+\mathrm{b}+\mathrm{c}+\mathrm{d} ;$ \\
\hline
\end{tabular}

then a multiple addition of intervals is described in the traditional mathematical notation. Besides the possibility of overloading operator symbols, one is allowed to use named operators. Such operators must be preceded by a priority declaration. There exist four different levels of priority, each represented by its own symbol:

- monadic $\quad$ : $\uparrow \quad$ level 3 (highest priority)

- multiplicative : * level 2

- additive $:+$ level 1

- relational : = level 0

For example, an operator for the calculation of the binomial coefficient $\left(\begin{array}{l}n \\ k\end{array}\right)$ can be defined in the following manner

priority choose $=* ; \quad\{$ priority declaration

operator choose (n,k: integer) binomial: integer;

var $i, r$ : integer;

begin

if $\mathrm{k}>\mathrm{n} \operatorname{div} 2$ then $\mathrm{k}:=\mathrm{n}-\mathrm{k}$;

$\mathrm{r}:=1$;

for $\mathrm{i}:=1$ to $\mathrm{k}$ do

$\mathrm{r}:=\mathrm{r} *(\mathrm{n}-\mathrm{i}+1) \operatorname{div} \mathrm{i}$;

binomial $:=r$;

end;

\begin{tabular}{|c|c|}
\hline mathematical notation & corresponding program statement \\
\hline$c:=\left(\begin{array}{l}n \\
k\end{array}\right)$ & $c:=\mathrm{n}$ choose $\mathrm{k}$ \\
\hline
\end{tabular}

The operator concept realized in PASCAL-XSC offers the possibilities of

- defining an arbitrary number of operators

- overloading operator symbols or operator names arbitrarily many times

- implementing recursively defined operators

The identification of the suitable operator depends on both the number and the type of the operands according to the following weighting-rule:

If the actual list of parameters matches the formal list of parameters of two different operators, then the one which is chosen has the first "better matching" parameter. "Better matching" means that the types of the operands must be consistent and not only conforming. 


\section{Example:}

operator $+*$ (a: integer; b: real) irres: real;

:

operator $+*(a$ : real; b: integer $)$ rires: real;

$\vdots$

var $\mathrm{x}$ : integer;

$\mathrm{y}, \mathrm{z}$ : real;

$\vdots$

$\mathrm{z}:=\mathrm{x}+* \mathrm{y} ; \Longrightarrow 1$. operator

$\mathrm{z}:=\mathrm{y}+* \mathrm{x} ; \Longrightarrow 2$. operator

$\mathrm{z}:=\mathrm{x}+* \mathrm{x} ; \Longrightarrow 1$. operator

$\mathrm{z}:=\mathrm{y}+* \mathrm{y} ; \Longrightarrow$ impossible!

Also, PASCAL-XSC offers the possibility to overload the assignment operator : = Due to this, the mathematical notation may also be used for assignments:

\section{Example:}

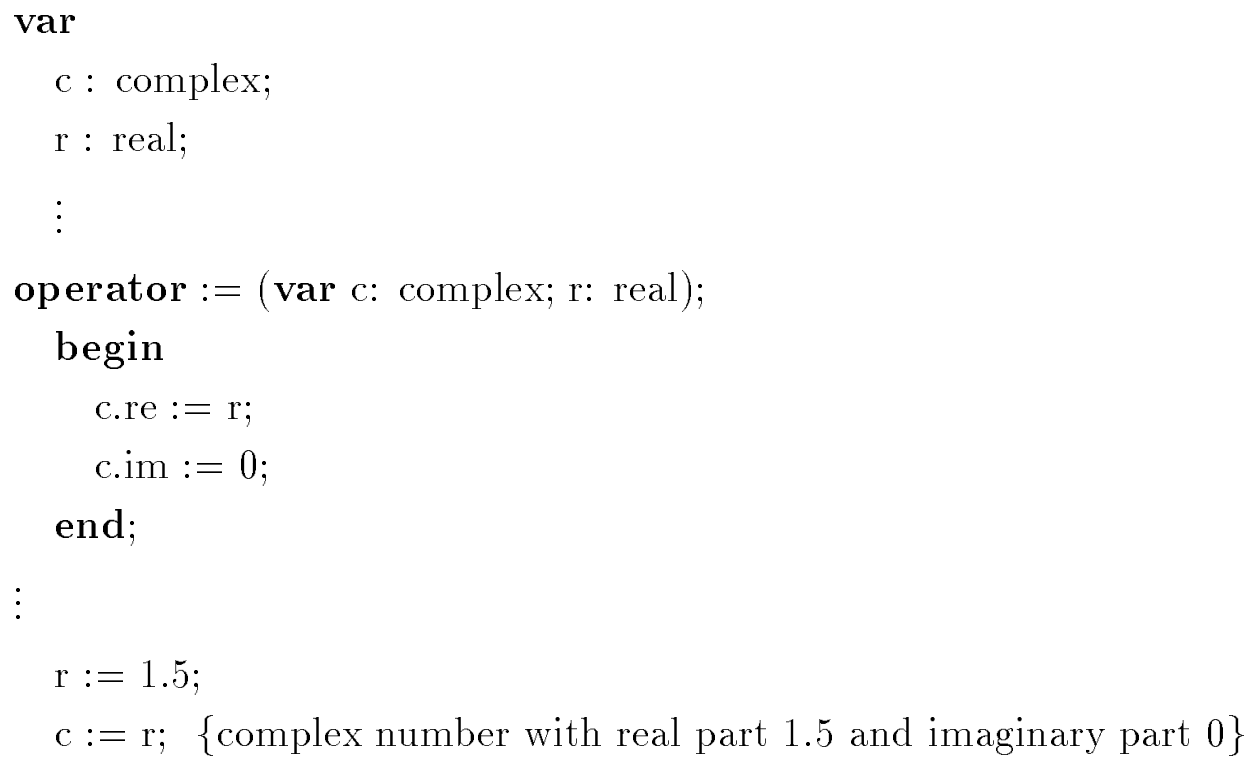

\subsection{Overloading of Subroutines}

Standard PASCAL provides the mathematical standard functions

sin, cos, arctan, exp, ln, sqr, and sqrt 
for numbers of type real only. In order to implement the sine function for interval arguments, a new function name like isin(...) must be used, because the overloading of the standard function name $\sin$ is not allowed in standard PASCAL.

By contrast, PASCAL-XSC allows overloading of function and procedure names, whereby a generic symbol concept is introduced into the language. So the symbols

$$
\text { sin, cos, arctan, exp, ln, sqr, and sqrt }
$$

can be used not only for numbers of type real, but also for intervals, complex numbers, and other mathematical spaces. To distinguish between overloaded functions or procedures with the same name, the number, type, and weighting of their arguments are used, similar to the method for operators. The type of the result, however, is not used.

\section{Example:}

$$
\begin{aligned}
& \text { procedure rotate (var a,b: real); } \\
& \text { procedure rotate (var a,b,c: complex); } \\
& \text { procedure rotate (var a,b,c: interval); }
\end{aligned}
$$

The overloading concept also applies to the standard procedures read and write in a slightly modified way. The first parameter of a new declared input/output procedure must be a var-parameter of file type and the second parameter represents the quantity that is to be input or output. All following parameters are interpreted as format specifications.

\section{Example:}

procedure write (var f: text; c: complex; w: integer);

\section{begin}

$$
\text { write (f, '(', c.re : w, ', c.im : w, ')'); }
$$

end

Calling an overloaded input/output procedure the file parameter may be omitted corresponding to a call with the standard files input or output. The format parameters must be introduced and seperated by colons. Moreover, several input or output statements can be combined to a single statement just as in standard PASCAL.

\section{Example:}

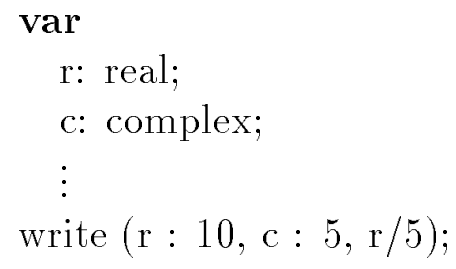




\subsection{The Module Concept}

Standard PASCAL basically assumes that a program consists of a single program text which must be prepared completely before it can be compiled and executed. In many cases, it is more convenient to prepare a program in several parts, called modules, which can then be developed and compiled independently of each other. Moreover, several other programs may use the components of a module without their being copied into the source code and recompiled.

For this purpose, a module concept has been introduced in PASCAL-XSC. This new concept offers the possibilities of

- modular programming

- syntax check and semantic analysis beyond the bounds of modules

- implementation of arithmetic packages as standard modules

Three new keywords have been added to the language:

$$
\begin{array}{ll}
\text { module } & : \text { starts a new module } \\
\text { global } & : \text { indicates items to be passed to the outside } \\
\text { use } & : \text { indicates imported modules }
\end{array}
$$

A module is introduced by the keyword module followed by a name and a semicolon. The body is built up quite similarly to that of a normal program with the exception that the word symbol global can be used directly in front of the keywords const, type, var, procedure, function, and operator and directly after use and the equality sign in type declarations.

Thus it is possible to declare private types as well as non-private types. The structure of a private type is not known outside the declaration module and can only be influenced by subroutine calls. If, for example, the internal structure as well as the name of a type is to be made global, then the word symbol global must be repeated after the equality sign. By means of the declaration

$$
\text { global type complex }=\text { global record re, im : real end; }
$$

the type complex and its internal structure as a record with components re and $i m$ is made global.

A private type complex could be declared by

$$
\text { global type complex }=\text { record re, im: real end; }
$$

The user who has imported a module with this private definition cannot refer to the record components, because the structure of the type is hidden inside the module. 
A module is built up according to the following pattern:

module $\mathrm{m} 1$;

use $<$ other modules $>$;

$<$ global and local declarations $>$

begin

$<$ initialization of the module $>$

end.

For importing modules with use or use global the following transitivity rules hold

M1 use M2 and M2 use global M3 $\Rightarrow$ M1 use M3.

but
M1 use M2 and M2 use M3
$\nRightarrow$ M1 use M3,

Example: Let a module hierarchy be built up by

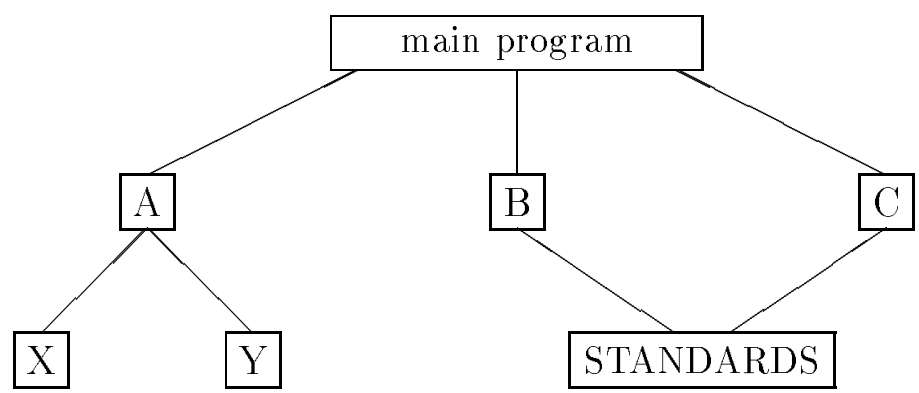

All global objects of the modules $\mathrm{A}, \mathrm{B}$, and $\mathrm{C}$ are visible in the main program unit, but there is no access to the global objects of $\mathrm{X}, \mathrm{Y}$ and STANDARDS. There are two possibilities to make them visible in the main program, too:

1. to write

use X, Y, STANDARDS

in the main program

2. to write

use global X, Y

in module $\mathrm{A}$ and

use global STANDARDS

in module B or C. 


\subsection{Dynamic Arrays}

In standard PASCAL there is no way to declare dynamic types or variables. For instance, program packages with vector and matrix operations can be implemented with only fixed (maximum) dimension. For this reason, only a part of the allocated memory is used if the user wants to solve problems with lower dimension only. The concept of dynamic arrays removes this limitation. In particular, the new concept can be described by the following characteristics:

- Dynamics within procedures and functions

- Automatic allocation and deallocation of local dynamic variables

- Economical employment of storage space

- Row access and column access to dynamic arrays

- Compatibility of static and dynamic arrays

Dynamic arrays must be marked with the word symbol dynamic. The great disadvantage of the conformant array schemes available in standard PASCAL is that they can only be used for parameters and not for variables or function results. So, this standard feature is not fully dynamic.

In PASCAL-XSC, dynamic and static arrays can be used in the same manner. At the moment, dynamic arrays may not be components of other data structures. The syntactical meaning of this is that the word symbol dynamic may only be used directly following the equality sign in a type definition or directly following the colon in a variable declaration. For instance, dynamic arrays may not be record components.

A two-dimensional array type can be declared in the following manner:

$$
\text { type matrix }=\text { dynamic array }[*, *] \text { of real; }
$$

It is also possible to define different dynamic types with corresponding syntactical structures. For example, it might be useful in some situations to identify the coefficients of a polynomial with the components of a vector or vice versa. Since PASCAL is strictly a type-oriented language, such structurally equivalent arrays may only be combined if their types have been previously adapted. The following example shows the definition of a polynomial and of a vector type (note that the type adaptation functions polynomial(...) and vector(...) are defined implicitly):

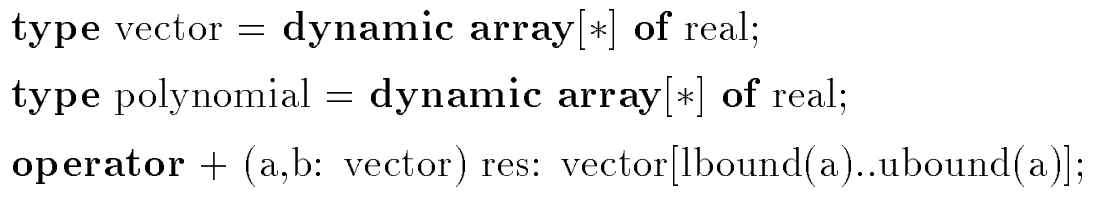




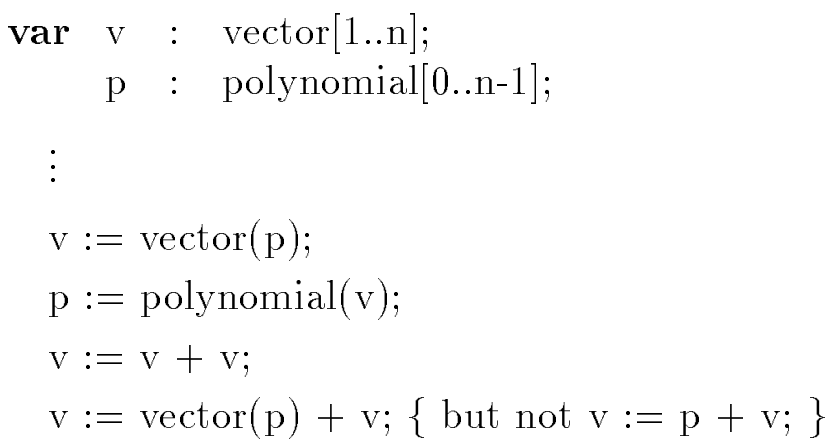

Access to the lower and upper index limits is made possible by the new standard functions 1 bound (...) and ubound(...), which are available with an optional argument for the index field of the designated dynamic variable. Employing these functions, the operator mentioned above can be written as

operator $+(a, b$ : vector $)$ res: vector $[\operatorname{lbound}(a) . . u b o u n d(a)]$;

var $\mathrm{i}$ : integer;

begin

for $\mathrm{i}:=\operatorname{lbound}(\mathrm{a})$ to ubound(a) do $\operatorname{res}[\mathrm{i}]:=\mathrm{a}[\mathrm{i}]+\mathrm{b}[\operatorname{lbound}(\mathrm{b})+\mathrm{i}-\operatorname{lbound}(\mathrm{a})]$

end;

Introduction of dynamic types requires an extension of the compatibility prerequisites. Just as in standard PASCAL, two array types are not compatible unless they are of the same type. Consequently, a dynamic array type is not compatible with a static type. In PASCAL-XSC value assignments are always possible in the cases listed in Table 5.

\begin{tabular}{|c|c|c|}
\hline Type of Left Side & Type of Right Side & Assignment Permitted \\
\hline anonymous dynamic & arbitrary array type & if structurally equivalent \\
known dynamic & known dynamic & if types are the same \\
anonymous static & arbitrary array type & if structurally equivalent \\
known static & known static & if types are the same \\
\hline
\end{tabular}

Table 5: Assignment Compatibilities

In the remaining cases, an assignment is possible only for an equivalent qualification of the right side (see [20] or [21] for details).

In addition to access to each component variable, PASCAL-XSC offers the possibility of access to entire subarrays. If a component variable contains an $*$ instead of an index expression, it refers to the subarray with the entire index range in the corresponding dimension, e. g. via $m[*, j]$ the $j$-th column of a two-dimensional array $m$ is accessed. This example demonstrates access to rows or columns of dynamic arrays: 


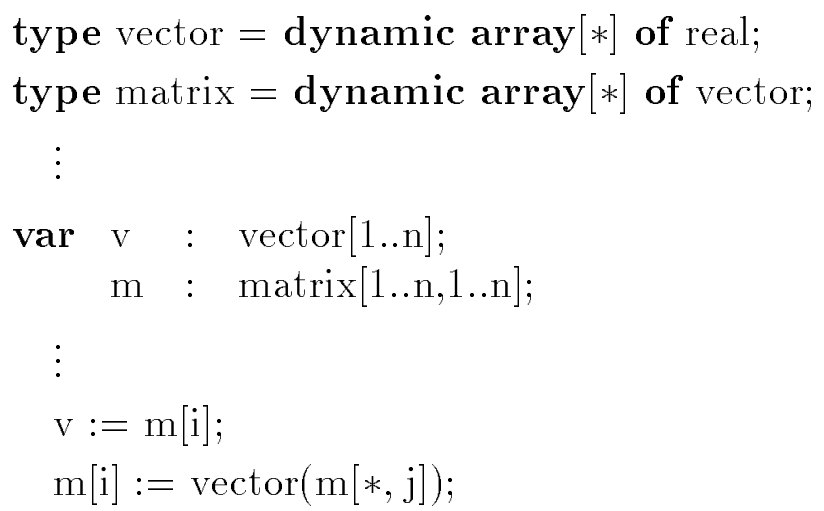

In the first assignment it is not necessary to use a type adaptation function, since both the left and the right side are of known dynamic type. A different case is demonstrated in the second assignment. The left-hand side is of known dynamic type, but the right-hand side is of anonymous dynamic type, so it is necessary to use the intrinsic adaptation function vector(...).

A PASCAL-XSC program which uses dynamic arrays should be built up according to the following scheme:

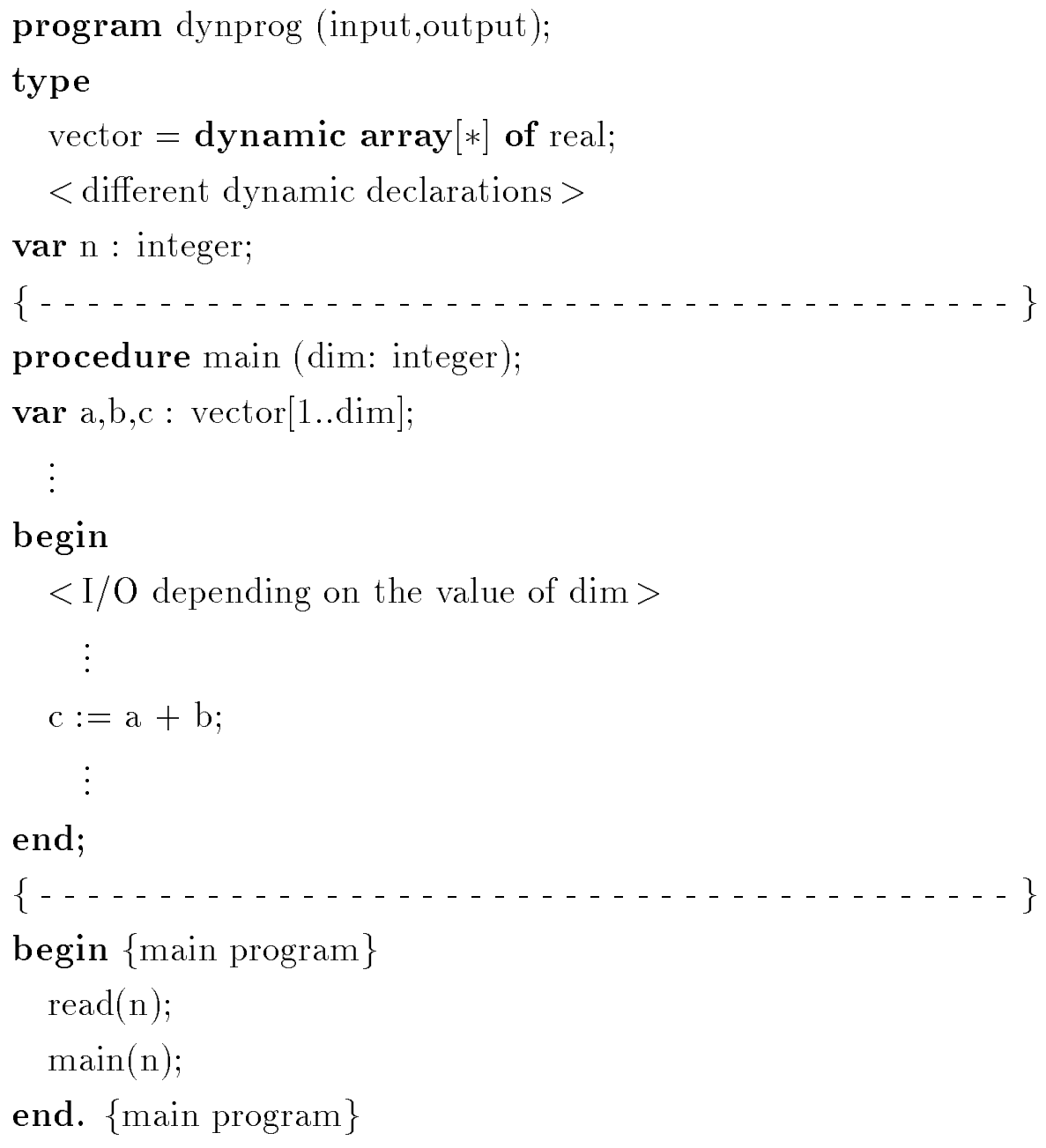

end. $\{$ main program $\}$ 
It is necessary to frame only the original main program by a procedure (here: main), which is refered to with the dimension of the dynamic arrays as a transfer parameter.

\subsection{Accurate Expressions}

The implementation of enclosure algorithms with automatic result verification or validation (see [17],[24],[28],[33]) makes extensive use of the accurate evaluation of dot products with the property (see [25])

$$
(\mathrm{RG}) \quad a \bigodot b:=\bigcirc \sum_{i=1}^{n} a_{i} \cdot b_{i}, \bigcirc \in\{\square, \triangle, \nabla\}, n \in \mathbb{N}
$$

To evaluate this kind of expression the new datatype dotprecision was introduced. This datatype accomodates the full floating-point range with double exponents (see [25],[24]). Based upon this type, so-called accurate expressions (\#-expressions), can be formulated by an accurate symbol (\#, \#*, \#<, \#>, or \#\#) followed by an exact expression enclosed in parentheses. The exact expression must have the form of a dot product expression and is evaluated without any rounding error. The following standard operations are available for dotprecision:

- conversion of real and integer values to dotprecision (\#)

- rounding of dotprecision values to real; in particular: downwardly directed rounding $(\#<)$, upwardly directed rounding $(\#>)$, and rounding to the nearest $(\# *)$

- rounding of a dotprecision expression to the smallest enclosing interval (\#\#)

- addition of a real number or the product of two real numbers to a variable of type dotprecision

- addition of a dot product to a variable of type dotprecision

- addition and subtraction of dotprecision numbers

- monadic minus of a dotprecision number

- the standard function sign returns $-1,0$, or +1 , depending on the sign of the dotprecision number

To obtain the unrounded or correctly rounded result of a dot product expression, the user needs to parenthesize the expression and precede it by the symbol \# which may optionally be followed by a symbol for the rounding mode. Table 6 shows the possible rounding modes with respect to the dot product expression form (see the appendix on page 41 for details). 


\begin{tabular}{|c|c|c|}
\hline Symbol & Expression Form & Rounding Mode \\
\hline \hline$\# *$ & scalar, vector or matrix & nearest \\
\hline$\#<$ & scalar, vector or matrix & downwards \\
\hline$\#>$ & scalar, vector or matrix & upwards \\
\hline$\# \#$ & scalar, vector or matrix & smallest enclosing interval \\
\hline$\#$ & scalar only & exact, no rounding \\
\hline
\end{tabular}

Table 6: Rounding Modes for Accurate Expressions

In practice, dot product expressions may contain a large number of terms making an explicit notation very cumbersome. To alleviate this difficulty in mathematics, the symbol $\sum$ is used. If for instance $A$ and $B$ are $n$-dimensional matrices, then the evaluation of

$$
\sum_{k=1}^{n} A(i, k) \cdot B(k, j)
$$

represents a dot product expression. PASCAL-XSC provides the equivalent shorthand notation sum for this purpose. The corresponding PASCAL-XSC statement for this expression is

$$
\mathrm{D}:=\#(\text { for } \mathrm{k}:=1 \text { to } \mathrm{n} \operatorname{sum}(\mathrm{A}[\mathrm{i}, \mathrm{k}] * \mathrm{~B}[\mathrm{k}, \mathrm{j}]))
$$

where $\mathrm{D}$ is a dotprecision variable.

Dot product expressions or accurate expressions are used mainly in computing a defect (or residual). In the case of a linear system $A x=b, A \in \mathbb{R}^{n \times n}, x, b \in \mathbb{R}^{n}$, as an example $A y \approx b$ is considered. Then an enclosure of the defect is given by $\diamond(b-A y)$ which in PASCAL-XSC can be realized by means of

$$
\# \#(\mathrm{~b}-\mathrm{A} * \mathrm{y})
$$

then there is only one interval rounding operation per component. To get verified enclosures for linear systems of equations it is necessary to evaluate the defect expression

$$
\vartheta(E-R A)
$$

where $R \approx A^{-1}$ and $E$ is the identity matrix. In PASCAL-XSC this expression can be programmed as

$$
\# \#(\mathrm{id}(\mathrm{A})-\mathrm{R} * \mathrm{~A})
$$

where an interval matrix is computed with only one rounding operation per component. The function $i d(.$.$) is a part of the module for real matrix/vector arithmetic$ generating an identity matrix of appropriate dimension according to the shape of A (see section 2.8). 


\subsection{The String Concept}

The tools provided for handling strings in standard PASCAL do not enable convenient text processing. For this reason, a string concept was integrated into the language definition of PASCAL-XSC which admits a comfortable handling of textual information and even symbolic computation. With this new data type string, the user can work with strings of up to 255 characters. In the declaration part the user can specify a maximum string length less than 255. Thus a string s declared by

var s: string[40];

can be up to 40 characters long. The following standard operations are available:

- concatenation

operator $+(a, b$ : string $)$ conc: string;

- actual length

function length(s: string): integer;

- conversion string $\rightarrow$ real

function rval(s: string): real;

- conversion string $\rightarrow$ integer

function ival(s: string): integer;

- conversion real $\rightarrow$ string

function image(r: real; width,fracs,round: integer): string;

- conversion integer $\rightarrow$ string

function image(i,len: integer): string;

- extraction of substrings

function substring(s: string; $i, j$ : integer $)$ : string;

- position of first appearance

function pos(sub,s: string): integer;

- relational operators $<=,<,>=,>,<>$, =, and in 


\subsection{Standard Modules}

The following standard modules are available:

- interval arithmetic (I_ARI)

- complex arithmetic ( $\left.\mathrm{C}_{-} \mathrm{ARI}\right)$

- complex interval arithmetic (CI_ARI)

- real matrix/vector arithmetic (MV_ARI)

- interval matrix/vector arithmetic (MVI_ARI)

- complex matrix/vector arithmetic (MVC_ARI)

- complex interval matrix/vector arithmetic (MVCI_ARI)

These modules may be incorporated via the use-statement described in section 2.4. As an example, Table 7 exhibits the operators provided by the module for interval matrix/vector arithmetic.

\begin{tabular}{|c|c|c|c|c|c|c|}
\hline $\begin{array}{r}\text { right } \\
\text { operand } \\
\text { left } \\
\text { operand }\end{array}$ & $\begin{array}{c}\text { integer } \\
\text { real }\end{array}$ & interval & rvector & ivector & rmatrix & imatrix \\
\hline monadic & & & &,+- & &,+- \\
\hline $\begin{array}{c}\text { integer } \\
\text { real }\end{array}$ & & & & * & & * \\
\hline interval & & & * & $*$ & * & * \\
\hline rvector & & $*, /$ & $+*$ & $\begin{array}{c}+*, \\
+,-, *, \\
\text { in },=,<>\end{array}$ & & \\
\hline ivector & $*, /$ & $*, /$ & $\begin{array}{c}+*, \\
+,-, *, \\
=,<>\end{array}$ & $\begin{array}{c}+*, * *, \\
+,-, *, \\
\text { in, }=,<>,><, \\
<=,<,>=,>\end{array}$ & & \\
\hline rmatrix & & $*, /$ & & * & $+*$ & $\begin{array}{c}+* \\
+,-, * \\
\text { in },=,<>\end{array}$ \\
\hline imatrix & $*, /$ & $*, /$ & * & * & $\begin{array}{c}+*, \\
+,-, *, \\
=,<>\end{array}$ & $\begin{array}{c}+*, * *, \\
+,-, *, \\
\text { in, }=,\langle>,\rangle<, \\
<=,\langle,\rangle=,\rangle\end{array}$ \\
\hline
\end{tabular}

Table 7: Predefined Arithmetical and Relational Operators of the Module MVI_ARI

In addition to these operators, the module MVI_ARI provides the following generically named standard operators, functions, and procedures

intval, inf, sup, diam, mid, blow, transp, null, id, read, and write.

The function intval is used to generate interval vectors and matrices, whereas inf and sup are selection functions for the infimum and supremum of an interval object. The diameter and the midpoint of interval vectors and matrices can be computed by diam and mid, blow yields an interval inflation, and transp delivers the transpose of a matrix. 
Zero vectors and matrices are generated by the function null, while id returns an identity matrix of appropriate shape. Finally, there are the generic input/outputprocedures read and write, which may be used in connection with all matrix/vector data types defined in the modules mentioned above.

\subsection{Problem-Solving Routines}

PASCAL-XSC routines for solving common numerical problems have been implemented. The applied methods compute a highly accurate enclosure of the true solution of the problem and, at the same time, prove the existence and the uniqueness of the solution in the given interval. The advantages of these new routines are listed in the following:

- The solution is computed with maximum or high, but always controlled accuracy, even in many ill-conditioned cases.

- The correctness of the result is automatically verified, i. e. an enclosing set is computed which guarantees existence and uniqueness of the exact solution contained in this set.

- In case, that no solution exists or that the problem is extremely illconditioned, an error message is indicated.

Particularly, PASCAL-XSC routines cover the following subjects:

- linear systems of equations

- full systems (real, complex, interval, cinterval)

- matrix inversion (real, complex, interval, cinterval)

- least squares problems (real, complex, interval, cinterval)

- computation of pseudo inverses (real, complex, interval, cinterval)

- band matrices (real)

- sparse matrices (real)

- polynomial evaluation

- in one variable (real, complex, interval, cinterval)

- in several variables (real)

- zeros of polynomials (real, complex, interval, cinterval)

- eigenvalues and eigenvectors

- symmetric matrices (real)

- arbitrary matrices (real, complex, interval, cinterval)

- initial and boundary value problems of ordinary differential equations

- linear

- nonlinear 
- evaluation of arithmetic expressions

- nonlinear systems of equations

- numerical quadrature

- integral equations

- automatic differentiation

- optimization

\section{The Implementation of PASCAL-XSC}

Since 1976, a PASCAL extension for scientific computation has been in the process of being defined and developed at the Institute for Applied Mathematics at the University of Karlsruhe. The PASCAL-SC compiler has been implemented on several computers (Z80, 8088, and 68000 processors) under various operating systems. This compiler has already been on the market for the IBM PC/AT and the ATARI-ST (see [22], [23]).

The new PASCAL-XSC compiler is now available for personal computers, workstations, mainframes, and supercomputers by means of an implementation in $\mathrm{C}$. Via a PASCAL-XSC-to-C precompiler and a runtime system implemented in $\mathrm{C}$, the language PASCAL-XSC may be used, among other systems, on all UNIX systems in an almost identical way. Thus, the user has the possibility to develop his programs for example on a personal computer and afterwards get them running on a mainframe via the same compiler.

A complete description of the language PASCAL-XSC and the arithmetic modules as well as a collection of sample programs is given in [20] and [21].

\section{PASCAL-XSC Sample Program}

In the following, a complete PASCAL-XSC program is listed, which demonstrates the use of some of the arithmetic modules. Employing the module LIN_SOLV, the solution of a system of linear equations is enclosed in an interval vector by succecsive interval iterations.

The procedure main, which is called in the body of lin_sys, is only used for reading the dimension of the system and for allocation of the dynamic variables. The numerical method itself is started by the call of procedure linear_system_solver defined in module LIN_SOLV. This procedure may be called with arbitrary dimension of the employed arrays.

For detailed information on iteration methods with automatic result verification see [17], [24], [28], or [32], for example. 


\section{Main Program}

program lin_sys (input,output);

\{ Program for verified solution of a linear system of equations. The $\}$ \{ matrix $A$ and the right-hand side $b$ of the system are to be read in. \{ The program delivers either a verified solution or a corresponding \} $\{$ failure message.

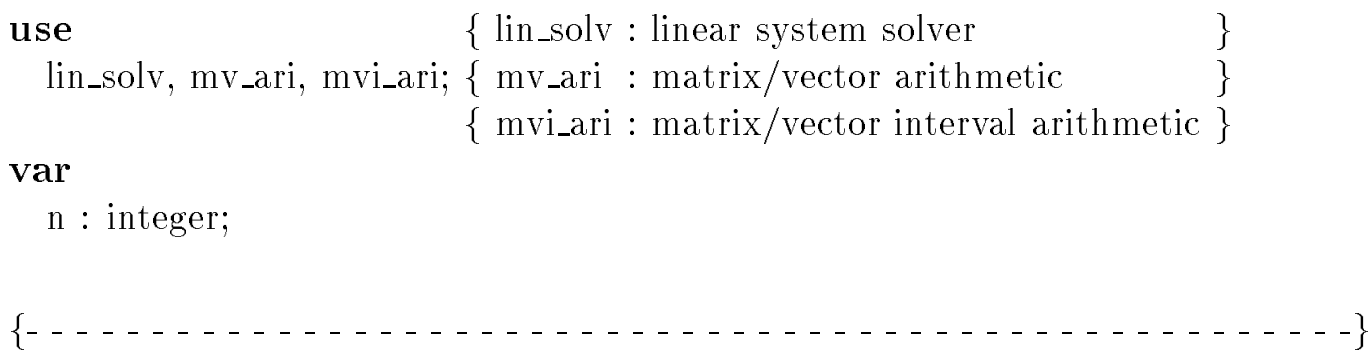

procedure main ( $\mathrm{n}$ : integer);

\{ The matrix A and the vectors $\mathrm{b}, \mathrm{x}$ are allocated dynamically with \{this subroutine being called. The matrix $\mathrm{A}$ and the right-hand side $\{b$ are read in and linear_system_solver is called.

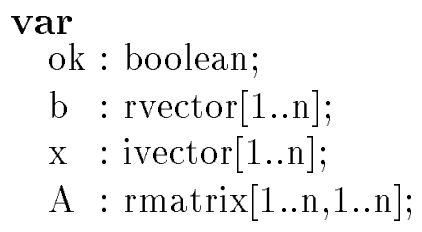

\section{begin}

writeln('Please enter the matrix A:'); $\operatorname{read}(\mathrm{A})$;

writeln('Please enter the right-hand side b:'); $\operatorname{read}(b)$;

linear_system_solver(A,b,x,ok);

\section{if ok then}

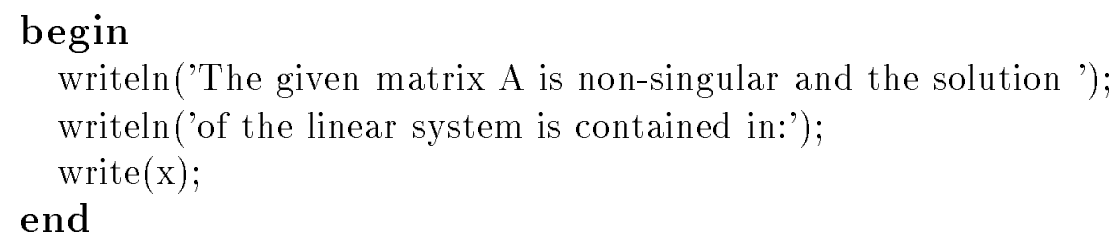


else

writeln ('No solution found !');

end; $\quad$ procedure main

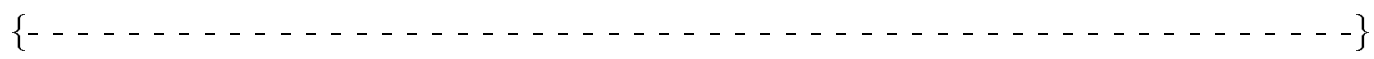

\section{begin}

write('Please enter the dimension $n$ of the linear system: ');

$\operatorname{read}(n)$;

main $(\mathrm{n})$;

end. $\{$ program lin_sys $\}$ 


\section{Module LIN_SOLV}

module lin_solv;

\{Verified solution of the linear system of equations $\mathrm{Ax}=\mathrm{b}$.

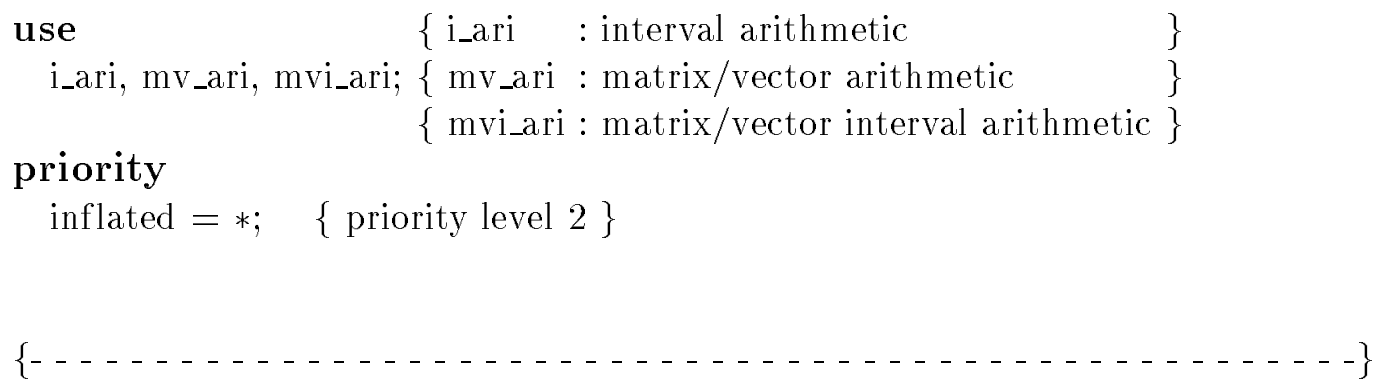

operator inflated (a : ivector; eps : real)infl: ivector[1..ubound(a)];

\{Computes the so-called epsilon inflation of an interval vector. $\}$

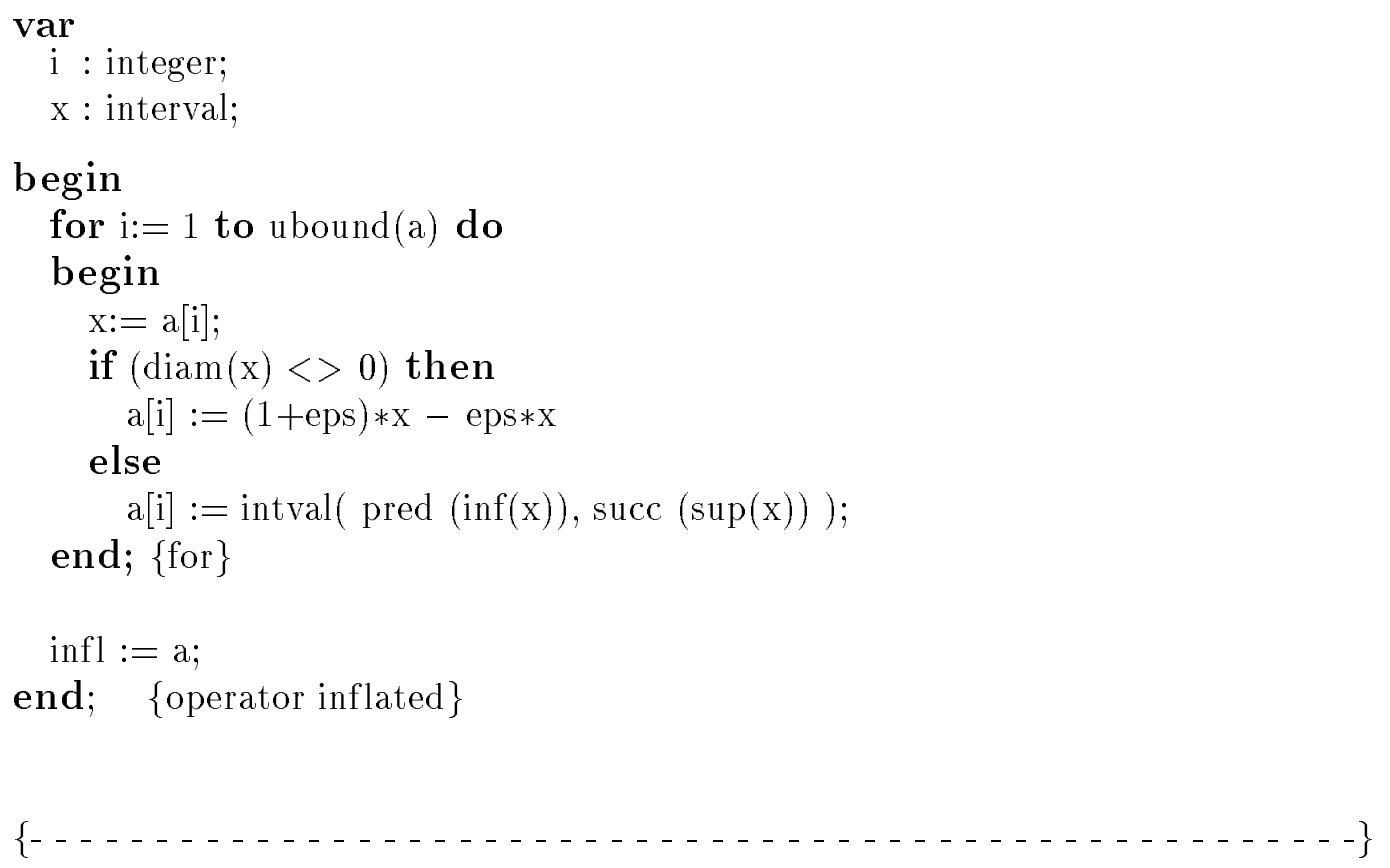


function approximate_inverse (A: rmatrix): rmatrix[1..ubound(A),1..ubound(A)];

$\{$ Computation of an approximate inverse of the $(n, n)$-matrix $A$ \}

$\{$ by application of the Gaussian elimination method.

var

$\mathrm{i}, \mathrm{j}, \mathrm{k}, \mathrm{n}$ : integer;

factor : real;

R, Inv, E : rmatrix[1..ubound(A),1..ubound(A)];

\section{begin}

$\mathrm{n}:=$ ubound $(\mathrm{A}) ; \quad\{$ dimension of $\mathrm{A}\}$

$\mathrm{E}:=\mathrm{id}(\mathrm{E}) ; \quad\{$ identity matrix $\}$

$\mathrm{R}:=\mathrm{A}$;

\{ Gaussian elimination step with unit vectors as \} \{right-hand sides. Division by $\mathrm{R}[\mathrm{i}, \mathrm{i}]=0$ indicates $\}$ $\{$ a probably singular matrix A.

for $\mathrm{i}:=1$ to $\mathrm{n}$ do

for $\mathrm{j}:=(\mathrm{i}+1)$ to $\mathrm{n}$ do

begin

factor $:=\mathrm{R}[\mathrm{j}, \mathrm{i}] / \mathrm{R}[\mathrm{i}, \mathrm{i}]$;

for $\mathrm{k}:=\mathrm{i}$ to $\mathrm{n}$ do $\mathrm{R}[\mathrm{j}, \mathrm{k}]:=\# *(\mathrm{R}[\mathrm{j}, \mathrm{k}]-$ factor $* \mathrm{R}[\mathrm{i}, \mathrm{k}])$;

$\mathrm{E}[\mathrm{j}]:=\mathrm{E}[\mathrm{j}]-$ factor $* \mathrm{E}[\mathrm{i}]$;

end; $\quad\{$ for $\mathrm{j}:=\ldots\}$

\{ Backward substitution delivers the rows of the inverse of $\mathrm{A}$.

for $\mathrm{i}:=\mathrm{n}$ downto 1 do

$\operatorname{Inv}[i]:=\# *(E[i]-$ for $k:=(i+1)$ to $n \operatorname{sum}(R[i, k] * \operatorname{Inv}[k])) / R[i, i]$

approximate_inverse $:=$ Inv;

end; \{function approximate_inverse

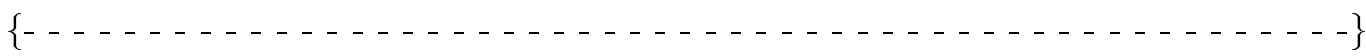


global procedure linear_system_solver (A : rmatrix; b : rvector;

var $\mathrm{x}$ : ivector; var ok : boolean);

\{Computation of a verified enclosure vector for the solution of the

\{linear system of equations. If an enclosure is not achieved after

\{ a certain number of iteration steps the algorithm is stopped and

$\{$ the parameter ok is set to false.

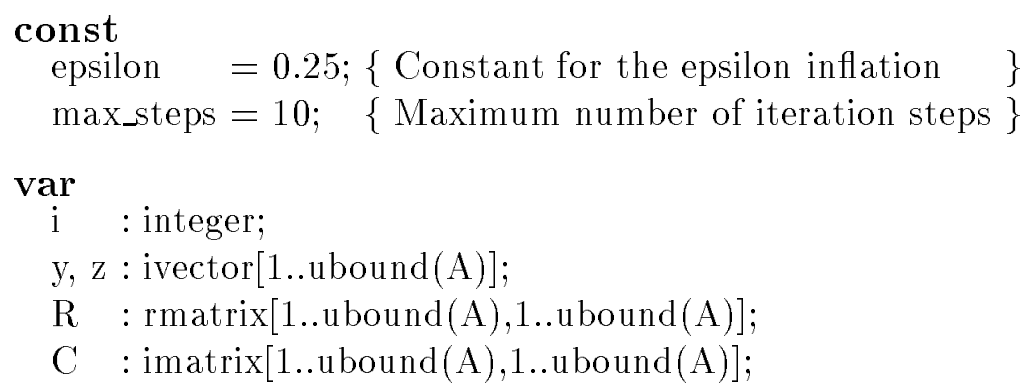

\section{begin}

$\mathrm{R}:=$ approximate_inverse $(\mathrm{A})$;

$\{\mathrm{R} * \mathrm{~b}$ is an approximate solution of the linear system and $\mathrm{z}$ is an enclosure $\}$ $\{$ of this vector. However, it does not usually enclose the true solution.

$\mathrm{z}:=\# \#(\mathrm{R} * \mathrm{~b})$;

\{An enclosure of $\mathrm{I}-\mathrm{R} * \mathrm{~A}$ is computed with maximum accuracy.

$\{$ The $(n, n)$ identity matrix is generated by the function call id $(A)$.

$\mathrm{C}:=\# \#(\mathrm{id}(\mathrm{A})-\mathrm{R} * \mathrm{~A})$;

$\mathrm{x}:=\mathrm{z} ; \quad \mathrm{i}:=0$

repeat

$$
\begin{aligned}
& \mathrm{i}:=\mathrm{i}+1 \text {; } \\
& \mathrm{y}:=\mathrm{x} \text { inflated epsilon; }\{\text { To obtain a true enclosure, the interval }\} \\
& \{\text { vector } c \text { is slightly enlarged. } \\
& \mathrm{x}:=\mathrm{z}+\mathrm{C} * \mathrm{y} ; \quad\{\text { The new iterate is computed. }\} \\
& \text { ok }:=\mathrm{x} \text { in } \mathrm{y} ; \quad\{\text { Is } \mathrm{c} \text { contained in the interior of } \mathrm{y} ?\}
\end{aligned}
$$

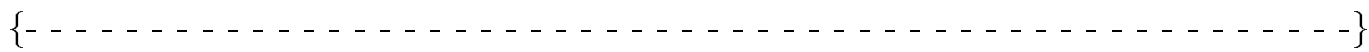

end. $\{$ module lin_solv 


\section{Appendix}

\section{Review of Real and Complex \#-Expressions}

\begin{tabular}{|c|c|c|}
\hline \#-Symbol & Result Type & Summands Permitted in the Exact Expression \\
\hline \# & dotprecision & $\begin{array}{l}\text { - variables, constants, and special function calls of } \\
\text { type integer, real, or dotprecision } \\
\text { - products of type integer or real } \\
\text { - scalar products of type real }\end{array}$ \\
\hline \multirow{6}{*}{$\begin{array}{l}\# * \\
\#< \\
\#>\end{array}$} & real & $\begin{array}{l}\text { - variables, constants, and special function calls of } \\
\text { type integer, real, or dotprecision } \\
\text { - products of type integer or real } \\
\text { - scalar products of type real }\end{array}$ \\
\hline & complex & $\begin{array}{l}\text { - variables, constants, and special function calls of } \\
\text { type integer, real, complex, or dotprecision } \\
\text { - products of type integer, real, or complex } \\
\text { - scalar products of type real or complex }\end{array}$ \\
\hline & rvector & $\begin{array}{l}\text { - variables and special function calls of type rvector } \\
\text { - products of type rvector (e.g. rmatrix * rvector, real } \\
\text { * rvector etc.) }\end{array}$ \\
\hline & cvector & $\begin{array}{l}\text { - variables and special function calls of type rvector } \\
\text { or cvector } \\
\text { - products of type rvector or cvector (e.g. cmatrix * } \\
\text { rvector, real * cvector etc.) }\end{array}$ \\
\hline & rmatrix & $\begin{array}{l}\text { - variables and special function calls of type rmatrix } \\
\text { - products of type rmatrix }\end{array}$ \\
\hline & cmatrix & $\begin{array}{l}\text { - variables and special function calls of type rmatrix } \\
\text { or cmatrix } \\
\text { - products of type rmatrix or cmatrix }\end{array}$ \\
\hline
\end{tabular}




\section{Review of Real and Complex Interval \#-Expressions}

\section{Syntax: \#\# ( Exact Expression )}

\begin{tabular}{|c|c|c|}
\hline \#-Symbol & Result Type & Summands Permitted in the Exact Expression \\
\hline \multirow{6}{*}{$\# \#$} & interval & $\begin{array}{l}\text { - variables, constants, and special function calls of } \\
\text { type integer, real, interval, or dotprecision } \\
\text { - products of type integer, real, or interval } \\
\text { - scalar products of type real or interval }\end{array}$ \\
\hline & cinterval & $\begin{array}{l}\text { - variables, constants, and special function calls of } \\
\text { type integer, real, complex, interval, cinterval, or } \\
\text { dotprecision } \\
\text { - products of type integer, real, complex, interval, or } \\
\text { cinterval } \\
\text { - scalar products of type real, complex, interval, or } \\
\text { cinterval }\end{array}$ \\
\hline & ivector & $\begin{array}{l}\text { - variables and special function calls of type rvector } \\
\text { or ivector } \\
\text { - products of type rvector or ivector }\end{array}$ \\
\hline & civector & $\begin{array}{l}\text { - variables and special function calls of type rvector, } \\
\text { cvector, ivector, or civector } \\
\text { - products of type rvector, cvector, ivector, or civector }\end{array}$ \\
\hline & imatrix & $\begin{array}{l}\text { - variables and special function calls of type rmatrix } \\
\text { or imatrix } \\
\text { - products of type rmatrix or imatrix }\end{array}$ \\
\hline & cimatrix & $\begin{array}{l}\text { - variables and special function calls of type rmatrix, } \\
\text { cmatrix, imatrix, or cimatrix } \\
\text { - products of type rmatrix, cmatrix, imatrix, or } \\
\text { cimatrix }\end{array}$ \\
\hline
\end{tabular}




\section{References}

[1] Allendörfer, U., Shiriaev, D.: PASCAL-XSC to C-A Portable PASCAL-XSC Compiler. In: [18], 91-104, 1991.

[2] Allendörfer, U., Shiriaev, D.: PASCAL-XSC - A portable development system. In [9], 1992.

[3] American National Standards Institute / Institute of Electrical and Electronic Engineers: $A$ Standard for Binary Floating-Point Arithmetic. ANSI/IEEE Std. 754-1985, New York, 1985.

[4] Bleher, J. H., Rump, S. M., Kulisch, U., Metzger, M., Ullrich, Ch., and Walter, W.: FORTRAN-SC: A Study of a FORTRAN Extension for Engineering/Scientific Computation with Access to ACRITH. Computing 39, 93 - 110, 1987.

[5] Bohlender, G., Grüner, K., Kaucher, E., Klatte, R., Krämer, W., Kulisch, U., Rump, S., Ullrich, Ch., Wolff von Gudenberg, J., and Miranker, W.: PASCAL-SC: A PASCAL for Contemporary Scientific Computation. Research Report RC 9009, IBM Thomas J. Watson Research Center, Yorktown Heights, New York, 1981.

[6] Bohlender, G., Grüner, K., Kaucher, E., Klatte, R., Kulisch, U., Neaga, M., Ullrich, Ch., and Wolff von Gudenberg, J.: PASCAL-SC Language Definition. Internal Report of the Institute for Applied Mathematics, University of Karlsruhe, 1985.

[7] Bohlender, G., Rall, L., Ullrich, Ch., and Wolff von Gudenberg, J.: PASCAL-SC: A Computer Language for Scientific Computation. Academic Press, New York, 1987.

[8] Bohlender, G., Rall, L., Ullrich, Ch. und Wolff von Gudenberg, J.: PASCAL-SC Wirkungsvoll programmieren, kontrolliert rechnen. Bibliographisches Institut, Mannheim, 1986 .

[9] Brezinsky, C. and Kulisch, U. (Eds): Computational and Applied Mathematics - Algorithms and Theory. Proceedings of the 13th IMACS World Congress, Dublin, Ireland. Elsevier, Science publishers B. V. To be published in 1992.

[10] Buchholz, W.: The IBM System/370 Vector Architecture. IBM Systems Journal 25/1, 1986.

[11] Cordes, D.: Runtime System for a PASCAL-XSC Compiler. In: [18], 151-160, 1991.

[12] Däßler, K. und Sommer, M.: PASCAL, Einführung in die Sprache. Norm Entwurf DIN 66256, Erläuterungen. Springer, Berlin, 1983.

[13] Hammer, R.: How Reliable is the Arithmetic of Vector Computers? In: [33], 1990.

[14] Hammer, R., Neaga, M., Ratz, D., Shiriaev, D.: PASCAL-XSC - A new language for scientific computing. (In Russian), Interval Computations 2, St. Petersburg, 1991,

[15] IBM High-Accuracy Arithmetic Subroutine Library (ACRITH). General Information Manual, GC 33-6163-02, 3rd Edition, 1986.

[16] IBM High-Accuracy Arithmetic Subroutine Library (ACRITH). Program Description and User's Guide, SC 33-6164-02, 3rd Edition, 1986.

[17] Kaucher, E., Kulisch, U., and Ullrich, Ch. (Eds.): Computer Arithmetic - Scientific Computation and Programming Languages. Teubner, Stuttgart, 1987.

[18] Kaucher, E., Markov, S. M., Mayer, G. (Eds): Computer Arithmetic, Scientific Computation and Mathematical Modelling. IMACS Annals on Computing and Applied Mathematics 12, J.C. Baltzer, Basel, 1991.

[19] Kirchner, R. and Kulisch, U.: Accurate Arithmetic for Vector Processors. Journal of Parallel and Distributed Computing 5, 250-270, 1988.

[20] Klatte, R., Kulisch, U., Neaga, M., Ratz, D. und Ullrich, Ch.: PASCAL-XSC Sprachbeschreibung mit Beispielen. Springer, Heidelberg, 1991. 
[21] Klatte, R., Kulisch, U., Neaga, M., Ratz, D. und Ullrich, Ch.: PASCAL-XSC Language Reference with Examples. Springer, Heidelberg, 1992.

[22] Kulisch, U. (Ed.): PASCAL-SC: A PASCAL Extension for Scientific Computation, Information Manual and Floppy Disks, Version ATARI ST. Teubner, Stuttgart, 1987.

[23] Kulisch, U. (Ed.): PASCAL-SC: A PASCAL Extension for Scientific Computation, Information Manual and Floppy Disks, Version IBM PC/AT (DOS). Teubner, Stuttgart, 1987.

[24] Kulisch, U. (Hrsg.): Wissenschaftliches Rechnen mit Ergebnisverifikation - Eine Einführung. Akademie Verlag, Ost-Berlin, Vieweg, Wiesbaden, 1989.

[25] Kulisch, U. and Miranker, W. L.: Computer Arithmetic in Theory and Practice. Academic Press, New York, 1981.

[26] Kulisch, U. and Miranker, W. L.: The Arithmetic of the Digital Computer: A New Approach. SIAM Review, Vol. 28, No. 1, 1986.

[27] Kulisch, U. and Miranker, W. L. (Eds.): A New Approach to Scientific Computation. Academic Press, New York, 1983.

[28] Kulisch, U. and Stetter, H. J. (Eds.): Scientific Computation with Automatic Result Verification. Computing Suppl. 6, Springer, Wien, 1988.

[29] Neaga, M.: Erweiterungen von Programmiersprachen für wissenschaftliches Rechnen und Erörterung einer Implementierung. Dissertation, Universität Kaiserslautern, 1984.

[30] Neaga, M.: PASCAL-SC - Eine PASCAL-Erweiterung für wissenschaftliches Rechnen. In: [24], 1989.

[31] Ratz, D.: The Effects of the Arithmetic of Vector Computers on Basic Numerical Methods. In: [33], 1990.

[32] Rump, S. M.: Solving Algebraic Problems with High Accuracy. In: [27], 1983.

[33] Ullrich, Ch. (Ed.): Contributions to Computer Arithmetic and Self-Validating Numerical Methods. J. C. Baltzer AG, Scientific Publishing Co., IMACS, 1990.

[34] Wolff von Gudenberg, J.: Einbettung allgemeiner Rechnerarithmetik in PASCAL mittels eines Operatorkonzeptes und Implementierung der Standardfunktionen mit optimaler Genauigkeit. Dissertation, Universität Karlsruhe, 1980. 